\section{Cobertura do Sistema de Vigilância Alimentar e Nutricional Indígena (SISVAN-I) e prevalência de desvios nutricionais em crianças Yanomami menores de 60 meses, Amazônia, Brasil}

\section{The coverage of the System for Nutrition Surveillance of Indigenous Peoples (SISVAN-I) and the prevalence of nutritional disorders in Yanomami children aged under 60 months, Amazonia, Brazil}

Lídia de Nazaré Pantoja 1

Jesem Douglas Yamall Orellana 2

Maurício Soares Leite 3

Paulo Cesar Basta 4

Programa de Pós-Graduação de Epidemiologia em Saúde Pública. Escola Nacional de Saúde Pública Sergio Arouca. Fundação Oswaldo Cruz. Rio de Janeiro, RJ, Brasil.

2 Instituto Leônidas e Maria Deane. Fundação Oswaldo Cruz. Rua Terezina, 476. Adrianópolis. Manaus, AM, Brasil. CEP. 69.057-070. Email: jesem_orellana@amazonia.fiocruz.br

3 Departamento de Nutrição. Universidade Federal de Santa Catarina. Florianópolis, SC, Brasil.

4 Escola Nacional de Saúde Pública Sergio Arouca. Fundação Oswaldo Cruz. Rio de Janeiro, RJ, Brasil.

\begin{abstract}
Objectives:to investigate the coverage of the System for Nutrition Surveillance of Indigenous People (SISVAN-I) and to estimate the prevalence of nutritional disorders in children aged under 60 months, in the Yanomami Special Sanitary District (DSEI-Yanomami).

Methods: a descriptive cross-sectional study was carried out between May 2008 and April 2009. Coverage was calculated by dividing the number of children evaluated by the total number of children enrolled. The health basic units (PB) with at least one record of weight and height available at each trimester, were included. The nutritional evaluation was based on the first record identified during the study period and used the World Health Organization curves as a point of reference (2006). Factors associated with low weight for age (W/A) were analyzed using logistic regression.

Results: the mean coverage at the eight $P B$ selected was $27.7 \%$. In all, $80.5 \%$ of children presented with low $\mathrm{H} / \mathrm{A} ; 57.5 \%$ with low W/A; $8.4 \%$ were thin and $5.5 \%$ overweight. The greatest likelihood of low W/A was found in children aged 36 to 59 months and in the Sanumá subgroup (OR=2.9 IC95\%: 2.1-3.9 and $O R=9.8$ IC95\%: 5.9-16.1, respectively). There was no difference between boys and girls.

Conclusions: although the coverage of the SISVAN-I has been low, the system has proved useful in revealing the severe nutritional situation among children in the DSEI-Yanomami and has signaled a need for intervention.
\end{abstract}

Key words Nutritional status, Health of indigenous peoples, Nutritional surveillance

\section{Resumo}

Objetivos: analisar a cobertura do Sistema de Vigilancia Alimentar e Nutricional Indígena (SISVANI) e estimar a prevalência de desvios nutricionais em crianças menores 60 meses, no Distrito Sanitário Especial Indígena Yanomami (DSEI-Yanomami).

Métodos: estudo transversal descritivo, efetuado entre maio/2008 e abril/2009. Para o cálculo da cobertura considerou-se a divisão das crianças avaliadas pelo total de crianças cadastradas e incluiramse os Pólos-Base com pelo menos um registro de peso e estatura em cada trimestre. A avaliação nutricional baseou-se no primeiro registro identificado no período em estudo e utilizou como referência as curvas da Organização Mundial da Saúde (2006). Fatores associados ao baixo peso para a idade (P/I) foram analisados por meio de regressão logística.

Resultados: a cobertura média nos 8 Pólos-Base selecionados foi 27,7\%. Ao todo, 80,5\% crianças apresentaram baixa E/I; 57,5\% baixo P/I; 8,4\% magreza e 5,5\% sobrepeso. Registraram-se as maiores chances de baixo P/I nas crianças 36 a 59 meses e no subgrupo Sanumá (OR=2,9; IC95\%: 2,13,9 e OR=9,8; IC95\%: 5,9-16,1, respectivamente). Não houve diferenças entre sexos.

Conclusões: embora a cobertura do SISVAN-I tenha sido baixa, o sistema mostrou-se útil ao revelar a grave situação nutricional das crianças do DSEIYanomami e sinalizar para a necessidade de medidas de intervenção.

Palavras-chave Estado nutricional, Saúde de populações indígenas, Vigilância nutricional 


\section{Introdução}

A partir das décadas de 1960 e 1970, respectivamente, a Codex Alimentarius Comission e a Organização das Nações Unidas para Alimentação e Agricultura (FAO) desenvolveram um vasto conjunto de normas e orientações voltadas ao monitoramento da segurança alimentar e nutricional de seus países membros, dentre as quais destaca-se a proposta da construção de sistemas de vigilância. ${ }^{1}$

O Brasil formalizou a criação do Sistema de Vigilância Alimentar e Nutricional (SISVAN), por intermédio da Portaria $\mathrm{n}^{\circ} 1.156$, de 31 de agosto de 1990. Entretanto, a ampliação da cobertura e o aperfeiçoamento do SISVAN só foram possíveis a partir da publicação da Portaria ${ }^{\circ} 710$, em 10 de junho de 1999.2 Neste período, os grandes inquéritos nacionais que se dedicavam ao estudo dos padrões alimentares e nutricionais do povo brasileiro não incluíam os povos indígenas como um segmento populacional específico em suas análises. 3 Isso significa que, até recentemente, o conhecimento sobre a situação de alimentação desses povos limitava-se aos dados obtidos a partir de estudos circunscritos a pequenas comunidades, em geral situadas na Amazônia Legal, onde se investigava grupos étnicos específicos. A despeito da limitada abrangência, os achados desses estudos eram quase unânimes em delinear um cenário amplamente desfavorável aos indígenas, no qual se registravam elevadas prevalências de déficits estaturais e ponderais e a ocorrência de anemia em proporções alarmantes, simultaneamente ao aumento crescente dos índices de sobrepeso. 4

Em 2006, reconhecendo-se a relevância e magnitude dos problemas de alimentação e nutrição entre os povos indígenas no Brasil e considerando o Sistema de Vigilância Alimentar e Nutricional Indígena (SISVAN-I) como uma das principais alternativas para o enfrentamento do problema, ${ }^{5}$ publicou-se a Portaria ministerial $n^{\circ} 984$ que regulamentou o SISVAN no âmbito do subsistema de atenção à saúde indígena.

Desde sua regulamentação, o SISVAN-I é considerado como importante fonte de dados relativos a alimentação e nutrição dos povos indígenas no Brasil. Entretanto, a escassez de dados sobre o tema continua sendo um desafio para os formuladores de políticas públicas. ${ }^{6}$

Ao longo dos últimos anos, o número de publicações científicas sobre o tema da alimentação e nutrição dos povos indígenas no Brasil vem aumentando acentuadamente, destacando-se a publicação do primeiro Inquérito Nacional sobre Saúde e
Nutrição dos Povos Indígenas, cuja coleta de dados ocorreu entre 2008-2009.7 Neste inquérito foram avaliadas mais de cinco mil crianças de 0 a 5 anos provenientes de 123 aldeias selecionadas por amostragem probabilística, com representatividade nas cinco macro-regiões brasileiras. Todavia, devido às características do desenho amostral, seus resultados não puderam ser extrapolados para qualquer Distrito Sanitário Especial Indígena (DSEI) ou para grupos étnicos em particular.

Apesar de atualmente haver mais informações sobre o estado nutricional da população indígena, ainda há uma diferença marcante em relação aos dados disponíveis para a população não indígena, sobretudo no tocante às análises que avaliam a tendência dos padrões nutricionais ao longo do tempo, em localidades selecionadas. Em um panorama onde a realização de grandes inquéritos ou até mesmo estudos de caso - enfrenta desafios de ordem logística e operacional, o SISVAN-I apresenta-se como um importante instrumento de monitoramento do estado nutricional e de avaliação das ações de saúde, em populações indígenas. Todavia, após quase uma década de sua criação, pouco se sabe acerca de aspectos relativos à estruturação, desempenho, cobertura e às possibilidades de análise desse sistema.

Os Yanomami constituem um conjunto cultural e linguístico composto por quatro subgrupos territorialmente adjacentes que falam línguas mutuamente inteligíveis, porém com características distintas: o Yanomam (que compreende aproximadamente 56\% da população), o Yanomami (ou Yanomae) (25\%), o Sanumá (14\%) e o Ninam (ou Yanam) (5\%). Pequena parte de seu território tradicional, situado no extremo noroeste de Roraima, é dividido com os vizinhos Ye'kuana ou Maiongong, pertencentes à família Karib. 8

A Terra Indígena Yanomami ocupa um território de 9.664.975 hectares, situado nas florestas tropicais do maciço guianense, em ambos os lados da fronteira entre o Brasil (Bacias do Alto Rio Branco e margem esquerda do Rio Negro) e a Venezuela (Bacias do Alto Orinoco e Cassiquiare). Este território é bastante acidentado, principalmente nas áreas próximas às serras Parima e Pacaraima, onde se tem a maior concentração Yanomami no Brasil. Nessa região, os solos são, em sua maioria, extremamente pobres e inadequados à agricultura intensiva. ${ }^{9}$

Os primeiros registros de contato dos Yanomami com a sociedade nacional remontam ao início do século XVIII. Entretanto, somente em meados do século XX, concretizou-se o contato permanente com a sociedade nacional, após a chegada de 
missões religiosas e a implantação de postos indígenas da Fundação Nacional do Índio (FUNAI). A partir desse momento, ocorreram mudanças expressivas no modo de vida desse povo, com a incorporação de utensílios metálicos e alimentos ocidentais e o consequente incentivo à instalação de alguns grupos no entorno dessas frentes de contato, resultando na sedentarização da população. ${ }^{10}$

Na década de 1980, iniciou-se a grande corrida do ouro em Roraima, período no qual dezenas de milhares de garimpeiros invadiram o território tradicional dos Yanomami, ${ }^{11}$ ocasionando intensa desorganização social, diversas epidemias, além da elevação da mortalidade por malária. Atualmente, a assistência à saúde dos Yanomami no Brasil é responsabilidade do Governo Federal por intermédio do Distrito Sanitário Especial Indígena Yanomami (DSEI-Yanomami), vinculado à Secretaria Especial de Saúde Indígena do Ministério da Saúde (SESAI/MS). A sede do DSEI-Yanomami encontrase em Boa Vista, capital de Roraima, local onde são coordenadas todas as ações em saúde. $\mathrm{Na}$ estrutura do DSEI-Yanomami, existem 37 Pólos-Base que operam como unidades básicas de saúde e prestam assistência a cerca de 19.000 indígenas das etnias Yanomami e Ye'kuana que residem em aproximadamente 272 aldeias, situadas entre os Estados do Amazonas e Roraima. 12

Considerando a relevância sociocultural e sanitária dessa etnia no cenário brasileiro, bem como a importância de se conhecer o perfil nutricional dessa população, este estudo teve como objetivo analisar a cobertura do SISVAN-I, bem como estimar a prevalência de desvios nutricionais em crianças indígenas menores 60 meses, no DSEIYanomami.

\section{Métodos}

Realizou-se um estudo transversal descritivo, a partir dos dados do SISVAN-I. Os dados foram extraídos do mapa de acompanhamento de crianças indígenas menores 60 meses, para o período de maio/2008 a abril/2009, e do módulo demográfico do Sistema de Informação da Atenção à Saúde Indígena (SIASI) do DSEI-Yanomami. Este período foi selecionado por coincidir com a fase de implantação do SISVAN-I no DSEI-Yanomami e com o período imediatamente posterior ao início do treinamento das equipes multidisciplinares de saúde indígena (EMSI), ambos sob a supervisão técnica da primeira autora (LNP). É importante assinalar que esses dados foram obtidos a partir dos registros impressos, uma vez que não estavam disponíveis em bancos de dados informati- zados.

Incluíram-se no estudo os Pólos-Base que reportaram ao menos um registro de peso e de estatura/ comprimento em menores 60 meses, nos trimestres que compreendem os períodos de $1^{\circ}$ de maio a 31 de julho de 2008; de $1^{\circ}$ de agosto a 31 de outubro de 2008; de $1^{\circ}$ de novembro de 2008 a 31 de janeiro de 2009; e de $1^{\circ}$ de fevereiro a 30 de abril de 2009, independentemente da data das aferições.

A exclusão dos registros duplicados foi efetuada conforme a proposta de Souza e Santos. 13

A análise da cobertura do SISVAN-I foi realizada mensalmente nos Pólos-Base. O percentual de cobertura foi calculado de acordo a Norma Técnica de Vigilância Alimentar e Nutricional para os Distritos Sanitários Especiais Indígenas, 14 utilizando a seguinte fórmula:

$\%$ de cobertura $=\mathrm{N}^{\circ}$ de crianças $<60$ meses acompanhadas no mês $\mathrm{X} 100$

$\mathrm{N}^{\circ}$ total de crianças $<60$ meses cadastradas no mês

As seguintes variáveis foram consideradas nas análises de cobertura e avaliação do estado nutricional da população de estudo: data de nascimento; sexo; data da aferição; peso; estatura ou comprimento; subgrupos indígenas; além dos Pólos-Base de residência.

A transformação dos valores antropométricos (estatura e peso) em escores z foi efetuada por meio do programa WHO-Anthro versão 3.2.2 (WHO, Department of Nutrition, Geneva, Switzerland). O conjunto de curvas de crescimento da Organização Mundial da Saúde15 foi utilizado como parâmetro de referência. Os índices estatura/idade (E/I), peso/idade $(\mathrm{P} / \mathrm{I})$ e peso/estatura ou comprimento $(\mathrm{P} / \mathrm{E})$ foram utilizados para avaliar o estado nutricional. Os índices E/I, P/I e P/E com valores de escore $\mathrm{z}<-2$ foram definidos como baixa estatura para a idade, baixo peso para a idade e baixo peso para a estatura (magreza), respectivamente. Valores de escore $\mathrm{z}>2$ para o índice $\mathrm{P} / \mathrm{E}$ foram classificados como sobrepeso.

Foram considerados biologicamente implausíveis ${ }^{16}$ valores $<-5$ ou $>3$ para o índice $\mathrm{E} / \mathrm{I} ;<-5$ ou $>5$ escore $\mathrm{z}$ para o índice $\mathrm{P} / \mathrm{I}$; e $<-4$ ou $>5$ escore $\mathrm{z}$ para o índice $\mathrm{P} / \mathrm{E}$, sendo portanto excluídos das análises.

O cálculo da prevalência dos desvios nutricionais segundo os índices de E/I, P/I e P/E foi realizado de acordo com as faixas etárias $(0,1$ a 5,9 meses; 6,0 a 11,9 meses; 12,0 a 23,9 meses; 24,0 a 35,9 meses; e 36,0 a 59,9 meses), tendo por base o primeiro registro antropométrico, correspondente ao 
período de estudo.

A associação entre os índices antropométricos e a variável faixa etária foi testada utilizando o teste de qui-quadrado de tendência linear. Em seguida, a associação bruta entre a variável dependente (baixo peso para a idade) e as variáveis independentes (sexo, subgrupo indígena e faixa etária) foi analisada utilizando o método de regressão logística simples. Variáveis associadas ao baixo peso para idade com nível de significância $<0,20$ foram incluídas no modelo de regressão múltipla. Essas variáveis foram incluídas em ordem decrescente de valores de $p$ e de acordo com os valores da razão de chances (Odds Ratio), com base no método stepwise forward. Variáveis que se mantiveram associadas ao desfecho, com nível de significância de $5 \%$ após ajuste pela variável sexo, foram mantidas do modelo final.

Os dados foram estruturados em planilhas eletrônicas do programa Microsoft Office Excel e analisados com auxílio do programa Statistical Package for the Social Sciences (SPSS) para Windows versão 17.0 (SPSS Inc., Chicago, IL, USA).

Este estudo foi aprovado pelo Comitê de Ética em Pesquisa da Escola Nacional de Saúde Pública Sergio Arouca (CEP/ENSP), sob Parecer No 224/11 e pela Comissão Nacional de Ética em Pesquisa do Conselho Nacional de Saúde (CONEP/CNS), sob Parecer $N^{0} 351 / 2012$, Protocolo de Pesquisa $n^{\circ}$ 16804 e CAAE $\mathrm{N}^{\circ}$ 0241.0.031.000-11. Além disto, contou com apoio e autorização da Fundação Nacional de Saúde (FUNASA), Coordenação Regional de Roraima (CORE/RR).

\section{Resultados}

Em oito $(21,6 \%)$ dos 37 PB existentes no DSEIYanomami foi possível encontrar ao menos um registro de peso e estatura em cada trimestre. Portanto, as análises relativas à cobertura deste estudo dizem respeito somente aos Pólos-Base Alto Catrimani; Aratha-ú; Auaris; Hakoma; Haxiu; Maloca Paapiu; Surucucu; e Waikás, todos situados no Estado de Roraima.

De acordo com a análise mensal das coberturas, a menor frequência de acompanhamento ocorreu em dezembro de 2008 e a maior em outubro de 2008, respectivamente. $\mathrm{Na}$ análise da cobertura ao longo dos 12 meses, observou-se que $27,7 \%$ das crianças foram acompanhadas mensalmente. O Pólo-Base Alto Catrimani foi o que apresentou a maior cobertura média e também a maior cobertura mensal, em maio de 2008. Já o Pólo-Base com menor cobertura média foi o de Aratha-ú. As menores coberturas mensais foram registradas nos Pólos-Base Maloca Paapiu e Hakoma, onde apenas uma criança foi avaliada em junho e dezembro de 2008, respectivamente. O Pólo-Base Surucucu foi o único que apresentou registros de acompanhamento mensal ininterruptos, ao longo de 10 meses (Tabela 1).

A quase totalidade dos dados antropométricos utilizados para calcular a prevalência dos desvios nutricionais foi coletada no primeiro trimestre de 2008, principalmente no mês de abril. Quanto à avaliação da plausibilidade dos valores dos escores $Z$, observou-se que um quinto dos registros não foram considerados biologicamente plausíveis para o índice E/I. (Tabela 2).

As prevalências de baixa E/I e de baixo P/I aumentaram progressiva e significativamente com a idade $(p<0,05)$. O sobrepeso foi observado em 5,5\% das crianças avaliadas, acometendo $16,8 \%$ dos menores de seis meses. O percentual de sobrepeso caiu gradativa e significativamente $(p<0,05)$ com a progressão da idade (Tabela 3).

Nas análises uni e multivariada não foram identificadas diferenças estatisticamente significativas nas prevalências de baixo $\mathrm{P} / \mathrm{I}$ entre sexos $\left(\mathrm{OR}_{\text {ajustada }}=1,1\right.$; IC95\%: 0,9-1,3). Por outro lado, verificou-se que os subgrupos Yanomam $\left(\mathrm{OR}_{\text {ajustada }}=8,0 ; \quad\right.$ IC95\%: 4,9-13,1), Ninan $\left(\mathrm{OR}_{\text {ajustada }}=5,2 ; \quad\right.$ IC95\%: 2,6-10,4) e Sanumá $\left(\mathrm{OR}_{\text {ajustada }}=9,8\right.$; IC95\%: 5,9-16,1) apresentaram aproximadamente oito, cinco e 10 vezes mais chances de apresentarem baixo P/I, quando comparados aos vizinhos Ye'kuana. Por sua vez, as chances de baixo $\mathrm{P} / \mathrm{I}$ aumentaram com a progressão da idade, variando de 1,6 vezes $\left(\mathrm{OR}_{\text {ajustada }}=1,6\right.$; IC95\%: 1,1-2,1) entre as crianças de 12 a 23 meses a 2,9 vezes $\left(\mathrm{OR}_{\text {ajustada }}=2,9\right.$; IC95\%: 2,1-3,9) nas crianças de 36 a 59 meses (Tabela 4$)$. 


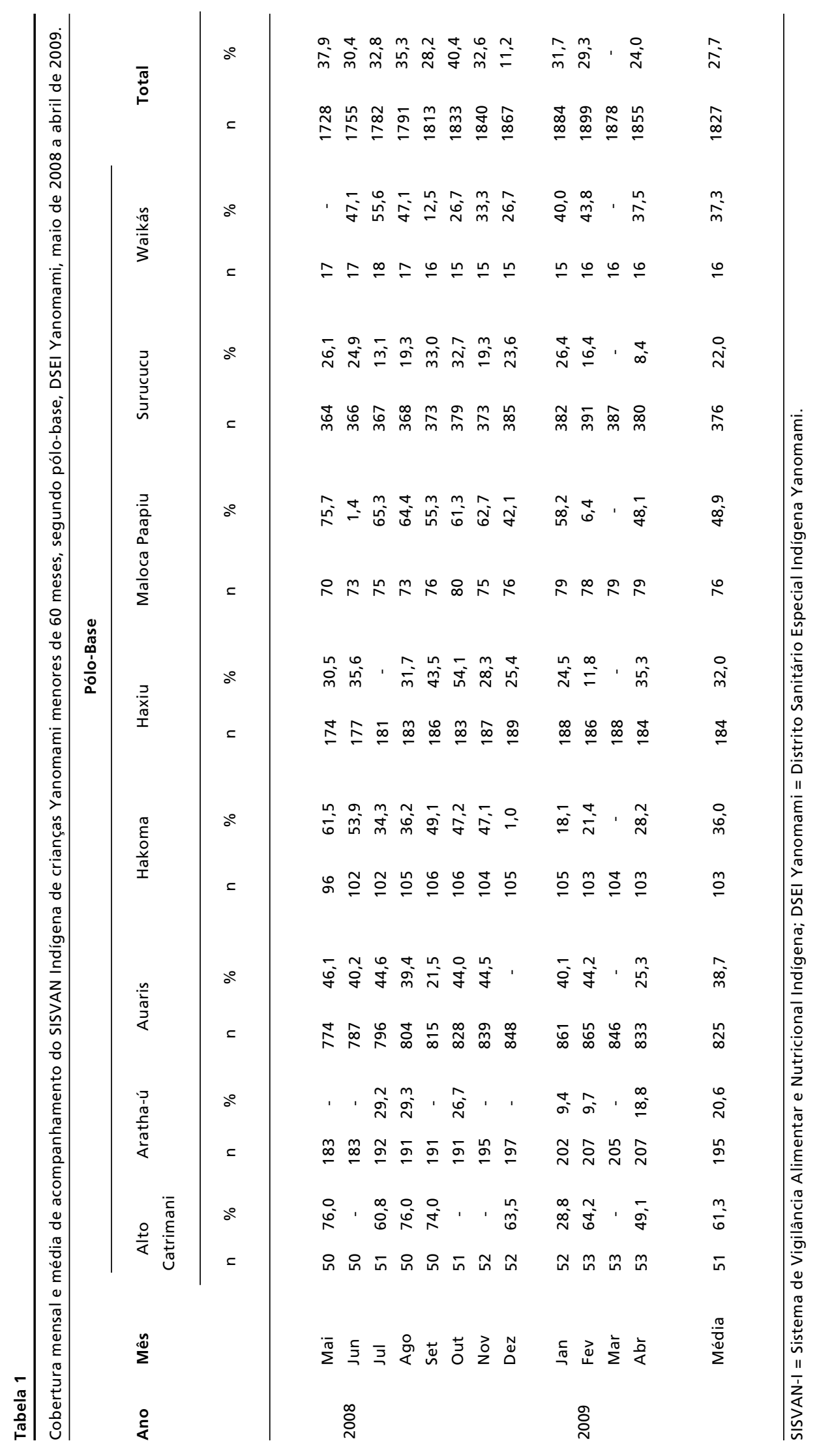


Distribuição de dados provavelmente implausíveis em crianças indígenas Yanomami menores de 60 meses, segundo faixa etária e índice antropométrico, DSEI Yanomami, maio de 2008 a abril de 2009.

\begin{tabular}{|c|c|c|c|c|c|c|}
\hline \multirow[t]{2}{*}{ Faixa etária (meses) } & \multicolumn{2}{|c|}{$E / I$} & \multicolumn{2}{|c|}{$P / I$} & \multicolumn{2}{|c|}{$\mathrm{P} / \mathrm{E}$} \\
\hline & $\mathrm{n}$ & $\%$ & $\mathrm{n}$ & $\%$ & $\mathrm{n}$ & $\%$ \\
\hline 0,1 a 5,9 & 316 & 20,9 & 316 & 7,6 & 310 & 4,2 \\
\hline 6,0 a 11,9 & 162 & 21,0 & 162 & 4,3 & 163 & 1,8 \\
\hline 12,0 a 23,9 & 339 & 20,1 & 339 & 5,3 & 339 & 2,4 \\
\hline 24,0 a 35,9 & 323 & 14,2 & 323 & 4,0 & 311 & 0,6 \\
\hline 36,0 a 59,9 & 527 & 19,5 & 527 & 8,9 & 514 & 1,6 \\
\hline Total & 1667 & 19,2 & 1667 & 6,1 & 1637 & 2,1 \\
\hline
\end{tabular}

DSEI Yanomami = Distrito Sanitário Especial Indígena Yanomami; E/I = estatura/idade; $\mathrm{P} / \mathrm{I}=$ peso/idade; $\mathrm{P} / \mathrm{E}=$ peso/estatura.

Distribuição de desvios nutricionais em crianças indígenas Yanomami menores de 60 meses, segundo faixa etária e índice antropométrico, DSEI-Yanomami, maio de 2008 a abril de 2009.

\begin{tabular}{|c|c|c|c|c|c|c|c|c|}
\hline \multirow[t]{3}{*}{ Faixa etária (meses) } & \multicolumn{2}{|c|}{$E / I^{a}$} & \multicolumn{2}{|c|}{$\mathrm{P} / \mathbf{I}^{\mathrm{a}}$} & \multicolumn{2}{|c|}{$\mathrm{P} / \mathrm{E}^{\mathrm{a}}$} & \multicolumn{2}{|c|}{ P/Ea } \\
\hline & \multicolumn{2}{|c|}{ Escores $Z<-2$} & \multicolumn{2}{|c|}{ Escores $Z<-2$} & \multicolumn{2}{|c|}{ Escores $Z<-2$} & \multicolumn{2}{|c|}{ Escores Z > 2} \\
\hline & $\mathrm{n}$ & $\%$ & $\mathrm{n}$ & $\%$ & $\mathrm{n}$ & $\%$ & $\mathrm{n}$ & $\%$ \\
\hline 0,1 a 5,9 & 250 & 65,2 & 292 & 43,2 & 297 & 6,1 & 297 & 16,8 \\
\hline 6,0 a 11,9 & 128 & 75,8 & 155 & 50,3 & 160 & 10,6 & 160 & 5,6 \\
\hline 12,0 a 23,9 & 268 & 79,5 & 321 & 54,2 & 331 & 9,1 & 331 & 3,3 \\
\hline 24,0 a 35,9 & 277 & 86,3 & 310 & 62,6 & 309 & 11,0 & 309 & 1,3 \\
\hline 36,0 a 59,9 & 424 & 87,7 & 480 & 67,5 & 506 & 6,9 & 506 & 2,8 \\
\hline Total & 1347 & $80,5^{*}$ & 1558 & $57,5^{*}$ & 1603 & 8,4 & 1603 & $5,5^{*}$ \\
\hline
\end{tabular}

DSEI Yanomami = Distrito Sanitário Especial Indígena Yanomami; a Teste $\chi^{2}$ de tendência linear; * $p<0,05$. 
Fatores sociodemográficos associados à ocorrência de baixo peso para a idade em crianças Yanomami menores de 60 meses, DSEI Yanomami, maio de 2008 a abril de 2009.

\begin{tabular}{|c|c|c|c|c|c|}
\hline \multirow[t]{2}{*}{ Variáveis } & \multicolumn{2}{|c|}{ Escores $Z<-2$} & \multirow[t]{2}{*}{ OR bruto (IC $80 \%)$} & \multirow[t]{2}{*}{ OR ajustado (IC95\%) } & \multirow[t]{2}{*}{$p$} \\
\hline & $\mathrm{n}$ & $\%$ & & & \\
\hline \multicolumn{6}{|l|}{ Subgrupo } \\
\hline Ye`kuana & 128 & 16,4 & 1,0 & 1,0 & \\
\hline Yanomam & 715 & 60,1 & $7,7(5,6-10,6)$ & $8,0(4,9-13,1)$ & 0,001 \\
\hline Ninan & 64 & 50,0 & $5,1(3,3-7,9)$ & $5,2(2,6-10,4)$ & 0,001 \\
\hline Sanumá & 651 & 63,4 & $8,8(6,4-12,2)$ & $9,8(5,9-16,1)$ & 0,001 \\
\hline \multicolumn{6}{|c|}{ Faixa etária (meses) } \\
\hline 0 a 5,9 & 292 & 43,2 & 1,0 & 1,0 & \\
\hline 6 a 11,9 & 155 & 50,3 & $1,3(1,0-1,7)$ & $1,3(0,9-1,9)$ & 0,236 \\
\hline 12 a 23,9 & 321 & 54,2 & $1,6(1,3-1,9)$ & $1,6(1,1-2,1)$ & 0,009 \\
\hline 24 a 35,9 & 310 & 62,6 & $2,2(1,8-2,7)$ & $2,4(1,7-3,4)$ & 0,001 \\
\hline 36 a 59,9 & 480 & 67,5 & $2,7(2,3-3,3)$ & $2,9(2,1-3,9)$ & 0,001 \\
\hline \multicolumn{6}{|l|}{ Sexo } \\
\hline Feminino & 754 & 56,9 & 1,0 & 1,0 & \\
\hline Masculino & 804 & 58,1 & $1,1(0,9-1,2)$ & $1,1(0,9-1,3)$ & 0,553 \\
\hline
\end{tabular}

DSEI Yanomami = Distrito Sanitário Especial Indígena Yanomami; A amostra incluída no modelo final foi de 1558 crianças.

\section{Discussão}

A partir deste estudo é possível avaliar que a cobertura do SISVAN-I foi muito baixa no DSEIYanomami, pois apenas $21,6 \%$ dos Pólos-Base (PB) existentes apresentavam ao menos um registro de peso e estatura por trimestre, entre maio de 2008 e abril de 2009. Embora as coberturas mensais médias nos Pólos-Base avaliados tenham oscilado entre $20 \mathrm{e}$ $61 \%$, apenas no PB Surucucu verificou-se acompanhamento mensal ininterrupto das crianças ao longo de um período de 10 meses.

O perfil antropométrico registrado entre os menores de cinco anos no DSEI-Yanomami exprime uma realidade precária, a qual expõe não só as desigualdades em saúde que afetam os Yanomami, como também a necessidade de medidas de intervenções que busquem melhorar as condições de vida e a assistência à saúde deste grupo.

A baixa cobertura do SISVAN-I registrada no DSEI-Yanomami traz consigo importantes implicações, dentre as quais se destacam a descontinuidade das ações ao longo do período analisado e a consequente impossibilidade de planejamento, acompanhamento e avaliação de possíveis medidas de intervenção - problemas que ganham especial significado em um cenário onde se registram elevadas prevalências de déficits nutricionais no segmento infantil. O conhecimento consistente, ampliado e continuado ao longo do tempo dos perfis alimentares e nutricionais e de seus determinantes, entre as crianças Yanomami, seria elemento fundamental para o delineamento de estratégias de intervenção custo-efetivas e adaptadas à realidade local.

O SISVAN-I é tido como a principal (em alguns contextos a única) fonte de informação sobre o acompanhamento nutricional de crianças indígenas no país. Portanto, o seu adequado funcionamento deveria ser útil não só para o correto diagnóstico do estado nutricional dessas crianças, como também, para que as famílias de crianças em risco nutricional tenham acesso a benefícios sociais, como os oriundos do Programa Bolsa Família. De acordo com as diretrizes do programa, o recebimento/ manutenção do benefício está sujeito a algumas condicionalidades, dentre as quais destacamos a de acompanhamento do crescimento e desenvolvimento das crianças menores de sete anos. ${ }^{17}$

Assim, o inadequado acompanhamento do estado nutricional das crianças indígenas pode resultar na suspensão de um benefício que deveria ser empregado para superação de situações de 
vulnerabilidade em segmentos da população brasileira que estão em situação de pobreza ou de extrema pobreza.

Informações da FUNASA indicam que, no período correspondente a este estudo, a entrada de dados no SISVAN-I vinha ocorrendo na maior parte dos DSEI, embora o grau de implantação do sistema variasse significativamente de um DSEI para o outro. ${ }^{18}$ Desde então, os órgãos gestores responsáveis pela atenção à saúde indígena não divulgaram mais qualquer relatório ou análise relativa ao funcionamento do SISVAN-I no país.

Desse modo, constata-se que o monitoramento contínuo do estado nutricional dos povos indígenas permanece como imenso desafio para gestores e executores do Subsistema de Atenção à Saúde Indígena e que a não informatização do SISVAN-I tem sido um dos possíveis entraves para o seu funcionamento. Cabe assinalar que dados referentes ao índice de $\mathrm{P} / \mathrm{I}$, disponíveis no SISVAN-Web, durante o período incluído em nossas análises, indicam que houve o acompanhamento de somente 20 crianças indígenas menores de cinco anos no Estado de Roraima. 19 Por outro lado, no Sistema de Gestão do Programa Bolsa Família19 havia o registro de cerca de 2000 crianças indígenas nesse mesmo período. Além da notória discrepância de dados entre o SISVAN-Web e o Sistema de Gestão do Programa Bolsa Família, percebe-se um claro subregistro também no Sistema de Gestão do Programa Bolsa Família, pois nos dois DSEI de Roraima a população-alvo ultrapassava 7000 crianças indígenas menores de cinco anos. 12 Além disso, evidenciam-se não somente os problemas de compatibilidade entre essas bases dados, como também a prioridade da inclusão de informações no Sistema de Gestão do Programa Bolsa Família em detrimento do SISVANWeb, conforme previamente demonstrado em outros contextos e regiões do país. ${ }^{20,21}$

Os resultados encontrados no DSEI-Yanomami constituem um exemplo das dificuldades (de ordem logística e administrativa) existentes para a implementação do SISVAN nas áreas indígenas. A despeito de suas singularidades, o DSEI-Yanomami pode ser tomado como ponto de partida para a discussão dessa temática em termos mais amplos.

No DSEI-Yanomami a quase totalidade das comunidades é geograficamente isolada, nas quais o acesso, só é possível após longas viagens de aeronaves de pequeno porte que levam as EMSI às sedes dos Pólos-Base. A partir de então só é possível chegar nas aldeias depois de horas de caminhada em meio à floresta amazônica. Em situações de emergência ou mesmo em campanhas de vacinação, as equipes de saúde necessitam utilizar helicópteros para terem acesso a essas populações. Além disso, na maioria das comunidades não existe estrutura mínima adequada para permanência das EMSI, fato que dificulta a realização de ações em saúde e o consequente acompanhamento de crianças com déficits nutricionais.

As visitas das EMSI aos Pólos-Base geralmente são realizadas uma vez ao mês e quando não há intercorrências a permanência dos profissionais é de no máximo sete dias. Este cenário impõe óbvios limites para o adequado acompanhamento da situação de saúde da população. A mobilidade de alguns grupos também impõe desafios adicionais, pois não raro famílias e às vezes comunidades inteiras se deslocam para outros locais, por motivos que incluem visitas a parentes e a obtenção de recursos naturais dispersos no território, tais como alimentos e materiais de construção para os domicílios, entre outros.

A limitada disponibilidade de equipamentos para aferição das medidas antropométricas e a inexistência, em boa parte dos PB, de espaços adequados para o exame das crianças podem ter comprometido a qualidade dos dados analisados. Embora todos os índices antropométricos tenham apresentado algum percentual de dados implausíveis, a maior proporção foi observada no índice $\mathrm{E} / \mathrm{I}$, correspondendo a um quinto dos registros. Essa proporção foi bastante expressiva, mesmo se considerarmos que a antropometria em crianças mais jovens (em especial o comprimento) exige mais cuidados e treinamento continuado. Todavia, problemas relativos à qualidade da coleta e registro de dados parecem não se restringir ao funcionamento do SISVAN em contextos indígenas. Em análise recente do sistema no Estado do Rio Grande do Sul, Damé et al.20 revelaram baixa concordância entre os diagnósticos realizados no Estado, frente aqueles observados na re-análise dos dados. Isto sinaliza que a expansão da cobertura corresponde a apenas um dos desafios para produção e análise dos dados do SISVAN-I.

Outro ponto que pode ter afetado a qualidade dos dados do SISVAN-I foi a elevada rotatividade dos profissionais que integram as EMSI do DSEI. Neste tipo de dinâmica, por vezes os profissionais mais qualificados, que já haviam sido treinados, são substituídos por outros sem o treinamento necessário para a coleta e a análise de dados antropométricos.

Destaca-se que em março de 2009 não houve qualquer registro de atividades relacionadas ao SISVAN-I nos Pólos-Base analisados. Nesse período houve uma paralisação das atividades, em decor- 
rência da quebra de contrato entre a Organização Não-Governamental prestadora de serviços de saúde e o órgão gestor do DSEI-Yanomami. Ao longo da última década, essa situação tornou-se recorrente no âmbito do Subsistema de Saúde Indígena, em todo o Brasil, pois havia a necessidade de que todos os contratos com as prestadoras de serviço fossem reavaliados antes de sua renovação ou cancelamento Os dados do SISVAN-I no DSEI-Yanomami constituem um exemplo claro das consequências negativas desta dinâmica estabelecida entre o órgão gestor e as organizações conveniadas.

Houve importante variação nas coberturas do sistema segundo Pólos-Base, tendo sido registradas as maiores coberturas no PB Alto Catrimani, especialmente no mês de maio, ocasião em que foi registrada cobertura de aproximadamente $80 \%$. Há de se destacar que o PB Alto Catrimani é administrado pela Diocese de Roraima desde a década de 1970 por meio de um convênio firmado com o Ministério da Saúde. É possível que a atuação dos missionários católicos junto a essa população, associada às melhores condições de acesso a esse PB, tenham contribuído para as maiores coberturas do SISVAN-I. Por outro lado, as menores coberturas foram registradas no Pólo-Base de Aratha-ú, que se situa em uma região de relevo acidentado, cercada por serras e rios não navegáveis, o que dificulta o acesso às aldeias. 22

Este estudo evidenciou as mais elevadas prevalências de baixa estatura para a idade em crianças indígenas $(80,5 \%)$ menores de 60 meses já reportadas na literatura nacional. Vale lembrar que entre as crianças examinadas essas prevalências aumentaram com a progressão da idade, atingindo valores próximos a $90 \%$ nas crianças de 36 a 59 meses. Frisa-se também que cerca de dois terços das crianças menores de seis meses apresentaram baixa estatura para a idade, sugerindo não só um problema nutricional recente, como também sua possível relação com o retardo de crescimento intra-uterino, decorrente, dentre outros aspectos, de uma assistência pré-natal insuficiente. ${ }^{23,24}$ Mesmo considerando-se as discussões sobre a adequação das curvas internacionais de crescimento para a avaliação de crianças indígenas, 15 os dados do DSEIYanomami são coerentes com o perfil epidemiológico caracterizado pelo predomínio de doenças infecciosas e parasitárias, onde as diarréias despontam como a primeira causa de morbidade entre os menores de cinco anos.

As prevalências de baixo peso para a idade foram igualmente elevadas nas crianças menores de 60 meses, tendo-se observado um aumento significa- tivo com o aumento da idade. A elevada prevalência de baixo peso para a idade, a partir dos seis meses, pode estar relacionada ao elevado percentual de crianças com baixa estatura. Ademais, a crescente exposição ambiental dessas crianças a microrganismos diversos pode ter igualmente favorecido o agravamento do seu estado nutricional. 4 Esses elevados níveis de desnutrição podem ter contribuído para a elevada taxa de mortalidade infantil, de 109,2 óbitos por mil nascidos vivos, 25 registrada no DSEI-Yanomami em 2010, uma das maiores entre todos os DSEI do país.

Em análise sobre a evolução do estado nutricional de crianças brasileiras menores de 60 meses, Batista Filho e Rissin 26 evidenciaram um notável declínio das prevalências de desnutrição ao longo das décadas de 1970, 1980 e 1990. Mais recentemente, na primeira década do século XXI, Monteiro et al. 27 confirmaram a tendência de redução dessas prevalências nas crianças brasileiras menores de 60 meses. No entanto, apesar da indiscutível redução da desnutrição na população geral de crianças brasileiras menores de sessenta meses, 28 não se deve ignorar que esse resultado pode não refletir a situação nutricional de segmentos específicos de nossa população. Os povos indígenas sequer foram considerados nas análises que apontam para estas tendências, na medida em que não foram incluídos nos grandes inquéritos nutricionais que as subsidiaram. Salvo exceções, não há dados que possibilitem, para este segmento da população brasileira, análises diacrônicas e a identificação de tendências. 4

A análise por meio da regressão logística revelou diferenças estatisticamente significativas na ocorrência de baixo peso para a idade entre os diversos subgrupos indígenas avaliados, sendo especialmente maiores as chances de baixo peso para a idade entre as crianças Sanumá. Esse achado tem implicações importantes para os serviços locais de saúde, já que evidencia a relevância dos diferentes contextos em sua determinação.

Um caso especialmente bem documentado é o dos Xavánte, em Mato Grosso, cujos perfis de saúde vêm sendo investigados desde os anos 1960.29 Dados de adultos Xavánte revelam que há uma progressiva diferenciação de perfis entre comunidades distintas, onde as transformações socioeconômicas e as relações com não indígenas exercem um papel preponderante na determinação do estado nutricional. Nas comunidades em que o contato resultou em um maior envolvimento com o mercado regional e com o trabalho remunerado, registram-se redução marcante dos níveis de atividade física e níveis alarmantes de excesso de peso. 30 
Além da elevada prevalência de magreza (baixa estatura para idade), outro dado que surpreendeu foi a destoante prevalência de sobrepeso entre os menores de seis meses $(16,8 \%)$, em relação as demais faixas etárias que apresentaram prevalências próximas a apenas três por cento. É possível que essa diferença seja consequência de problemas na coleta desses dados, especialmente de dificuldades técnicas envolvidas na mensuração do comprimento dos menores de seis meses.

A despeito da relevância de nossos achados para os formuladores de políticas em saúde pública, tanto no que diz respeito às falhas na cobertura do SISVAN-I, quanto à magnitude dos déficits nutricionais descritos, os resultados aqui apresentados devem ser interpretados com cautela. A análise da cobertura pautou-se em apenas oito $\mathrm{PB}$, de um universo de 37 existentes no DSEI. Todavia, vale lembrar que os demais $\mathrm{PB}$ não foram incluídos no estudo porque não havia ao menos um registro disponível a cada trimestre em análise. Por si, esse dado indiretamente informa sobre as dificuldades enfrentadas pelas EMSI para avaliar e monitorar o estado nutricional das crianças Yanomami.

Outro ponto a ser considerado diz respeito ao perfil nutricional delineado a partir dos dados antropométricos. Embora os déficits aqui registrados tenham sido os mais elevados já descritos na literatura especializada, não é possível extrapolar nossos achados para a totalidade das crianças Yanomami em nenhum dos períodos avaliados, em função das importantes limitações na cobertura do SISVAN. Além disso, somam-se aos registros ausentes um elevado percentual de casos considerados biologicamente implausíveis, em especial para o índice estatura/idade. Além de refletirem os problemas relativos à coleta e ao registro de dados, os dados faltantes impossibilitaram o diagnóstico nutricional da totalidade das crianças avaliadas no escopo deste estudo.

Contudo, vale ressaltar que mesmo considerando o elevado percentual de dados implausíveis e a baixa

\section{Referências}

1. Tritscher A, Miyagishima K, Nishidab C, Branca F Ensuring food safety and nutrition security to protect consumer health: 50 years of the Codex Alimentarius Commission. Bull World Health Organ. 2013; 91: 468-68A.

2. Coutinho JG, Cardoso AJC, Toral N, Silva ACF, Ubarana JA, Aquino KKNC, Nilson EAF, Fagundes A, Vasconcelos $\mathrm{AB}$. A organização da vigilância alimentar e nutricional no Sistema Único de Saúde: histórico e desafios atuais. Rev Bras Epidemiol. 2009; 12: 688-99. cobertura do SISVAN-I a análise incluiu uma população total de aproximadamente 1500 crianças indígenas. Embora essa amostra represente apenas parte das crianças Yanomami menores de cinco anos elegíveis para o estudo, com exceção do primeiro inquérito nacional de saúde e nutrição indígenas, a nossa amostra é a mais ampla já investigada em Terras Indígenas no Brasil.

Por fim, as especificidades envolvidas na atenção à saúde indígena, e que resultaram na criação do Subsistema de Atenção à Saúde para os Povos Indígenas no Brasil, de certo modo tornam mais complexa a concretização da proposta de monitoramento contínuo das condições de alimentação e nutrição deste segmento da população brasileira. Elas exigem investimentos em diversos aspectos para o alcance dos objetivos propostos - equipamentos, qualificação permanente de profissionais indígenas e não indígenas, logística em regiões de difícil acesso, além de garantia de continuidade na assistência à saúde.

Por outro lado, a literatura aponta para um maior potencial de sucesso do SISVAN-I quando comparado ao sistema desenvolvido entre não indígenas, em função do caráter circunscrito dessa população a determinadas áreas geográficas e a ampla cobertura do Subsistema de Atenção à Saúde Indígena. 6 Este potencial, associado à reconhecida importância do monitoramento contínuo de dados sobre nutrição e saúde, evidencia a necessidade de novas investigações com a finalidade de identificar e analisar nuances locais no funcionamento do SISVAN-I. Desta forma, acreditamos que o conhecimento produzido sobre o SISVAN pode contribuir para o alcance dos objetivos mais amplos do sistema.

\section{Agradecimentos}

Aos trabalhadores do DSEI-Yanomami, à Chefia e à Coordenação técnica do DSEI pelo cordial acesso aos dados.

3. Santos RV, Coimbra Jr. CEA. Cenários e tendências da saúde e da epidemiologia dos povos indígenas do Brasil. In: Coimbra Jr. CEA, Santos RV, Escobar AL, organizadores. Epidemiologia e saúde dos povos indígenas no Brasil. Rio de Janeiro: Fiocruz; 2003. p. 13-47.

4. Leite MS, Santos RV, Coimbra Jr. CEA, Gugelmin SA. Alimentação e nutrição dos povos indígenas no Brasil. In: Kac G, Sichieri R, Gigante D, organizadores. Epidemiologia nutricional. Rio de Janeiro: Fiocruz/Atheneu; 2007. p. 503-18. 
5. Caldas ADR, Santos RV. Vigilância alimentar e nutricional para os povos indígenas no Brasil: análise da construção de uma política pública em saúde. Physis. 2012; 22: 545-65.

6. Leite MS. Nutrição e alimentação em saúde indígena: notas sobre a importância e a situação atual. In: Garnelo L, Pontes AL, organizadores. Saúde Indígena: uma introdução ao tema. Brasília: MEC/ SECADI; 2012. p. 156-83

7. Coimbra Jr CEA, Santos RV, Welch JR, Cardoso AM, Souza MC, Garnelo M, Rassi E, Follér M, Horta BL. The first national survey of indigenous people's health and nutrition in Brazil: rationale, methodology, and overview of results. BMC Public Health. 2013; 13: 52

8. Albert B, Gomez GG. Saúde Yanomami: um manual etnolinguistico. Belém: Museu Paraense Emílio Goeldi; 1997.

9. Magalhães ED, Cavalcanti L. Morbi-Mortalidade Yanomami - 1991 a 1997. Boa Vista, RR: UFRR/FIOCRUZ; 1998.

10. Albert, B. Temps Du sang, temps des cendres. Représentation de la maladie, système rituel et space politique chez lês Yanomami Du sud-est. [tese]. Paris: Universidade de Paris X- Nanterre; 1985.

11. Nilsson MST. Organização indígena Yanomami: das ameaças ao seu território à representação política numa sociedade sem Estado. Agraria. 2008; 9: 25-43.

12. RENISI/ FUNASA (Rede Nacional de Pesquisas em Saúde dos Povos Indígenas/ Fundação Nacional de Saúde). 2013. Distritos Sanitários (Pirâmides Demográficas). [acesso em 29 ago 2013]. Disponível em: http://sis.funasa.gov.br/portal/mapa_dsei.asp

13. Souza LG, Santos RV. Componente Demográfico do Sistema de Informação da Atenção à Saúde Indígena, DSEI Xavánte, Mato Grosso, Brasil. Caderno CRH. 2009; 22 523-9.

14. Brasil. Ministério da Saúde. Fundação Nacional de Saúde. Vigilância alimentar e nutricional para os distritos sanitários especiais indígenas: Norma Técnica. Brasília, DF; 2006

15. WHO (World Health Organization). WHO child growth standards: length/height-for-age, weight-for-age, weightfor-length, weight-for-height and body mass index-for-age Methods and development. WHO (nonserial publication). Geneva; 2006

16. WHO (World Health Organization). Physical status: the use and interpretation of anthropometric indicators of nutritional status. Geneve; 1995.

17. Brasil. Ministério do Desenvolvimento Social. Portal do Bolsa Família. Condicionalidades. [Internet]. 2013. [acesso em 14 nov 2013]. Disponível em: http://www.mds.gov.br/bolsafamilia/condicionalidades

18. Brasil. Ministério da Saúde. Fundação Nacional de Saúde. Projeto Vigisus II. Relatório Subcomponente 2 Vigilância Alimentar e Nutricional. $2^{\circ}$ semestre de 2009. Brasília, DF; 2009 .
19. Brasil. Ministério da Saúde. Portal do Departamento de Atenção Básica. Estado nutricional dos indivíduos acompanhados por período, fase do ciclo da vida e índice. [Internet]. 2008-2009. [acesso em 2 jul 2013]. Disponível em: http://dabsistemas.saude.gov.br/sistemas/sisvan/relatorios_publicos/

20. Damé PKV, Pedroso MRO, Marinho CL, Gonçalves VM, Duncan BB, Fisher PD, Romero ALC, Castro TG. Sistema de Vigilância Alimentar e Nutricional (SISVAN) em crianças do Rio Grande do Sul, Brasil: cobertura, estado nutricional e confiabilidade dos dados. Cad Saúde Pública. 2011; 27: 2155-65.

21. Ferreira CS, Cherchiglia ML, César CC. O Sistema de Vigilância Alimentar e Nutricional como instrumento de monitoramento da Estratégia Nacional para Alimentação Complementar Saudável. Rev Bras Saúde Matern Infant. 2013; 13: 167-77.

22. URIHI Saúde Yanomami. Pólo-base Aratha-ú. O trabalho da URIHI na área Yanomami (2000-2004). Informações gerais sobre as regiões assistidas. [acesso em 24 março 2012]. Disponível em: http://www.urihi.org.br/info.html

23. Saenger P, Czernichow P, Hughes I, Reiter EO. Small for gestational age: short stature and beyond. Endocr Rev. 2007; 28: 219-51

24. Longo S, Bollani L, Decembrino L, Di Comite A, Angelini M, Stronati M. Short-term and long-term sequelae in intrauterine growth retardation (IUGR). J Matern Fetal Neonatal Med. 2013; 26: 222-5.

25. FUNASA (Fundação Nacional de Saúde). Relatório de gestão 2010: SUEST/RR. Boa Vista, RR; 2011. [acesso em 10 nov 2013]. Disponível em: http://www.funasa.gov.br/ site/wp-content/uploads/2011/10/rr.pdf

26. Batista Filho M, Rissin A. A transição nutricional no Brasil, tendências regionais e temporais. Cad Saúde Pública. 2003; 19: 181-91

27. Monteiro CA, Benicio MH, Konno SC, Silva AC, Lima AL, Conde WL. Causes for the decline in child under-nutrition in Brazil, 1996-2007. Rev Saúde Pública. 2009; 43: 35-43.

28. Victora CG, Barreto ML, Carmo Leal M, Monteiro CA, Schmidt SI, Paim J, Bastos FI, Almeida C, Travassos C, Reichenheim M, Barros FC. Health conditions and healthpolicy innovations in Brazil: the way forward. Lancet. 2011; 377: 32-46

29. Coimbra Jr. CEA, Flowers NM, Salzano FM, Santos RV. The Xavánte in transition: health, ecology, and bioanthropology in central Brazil. Michigan: University of Michigan Press; 2002.

30. Gugelmin AS, Santos RV. Ecologia humana e antropometria nutricional de adultos Xavánte, Mato Grosso, Brasil. Cad Saúde Pública. 2001; 17: 313-22.

Recebido em 10 de setembro de 2013

Versão final apresentada em 9 de dezembro de 2013

Aprovado em 27 de dezembro de 2013 